\title{
EFFECTIVE RADIAL DIFFUSIVITIES TO MODEL ENHANCED MASS AND HEAT TRANSFER IN LAMINAR FLOW REACTORS
}

\author{
J.A.T.A. DANTAS, A.O. MORAIS; J.A.W. GUT \\ University of São Paulo, Escola Politécnica, Department of Chemical Engineering \\ E-mail: jorgewgut@usp.br
}

\begin{abstract}
Main features of a laminar flow reactor (LFR) are streamline flow and parabolic velocity profile. Consequently, heating and cooling are difficult and there is a large spread in the residence time distribution (RTD). Enhanced heat and mass transfer can be found in coils or corrugated tubes or in the presence of wall roughness, curves, pipe fittings or mechanical vibration. Modeling these cases can be complex because of the induced secondary flow. Objective of this work is to introduce this enhanced transport in the LFR model by means of effective radial diffusivities of heat and mass. Model validation experiments were conducted in a LFR with high wall relative roughness and curves. RTD experiments were compared with model simulations of tracer dispersion to obtain the mass diffusivity. Heat transfer experiments provided the effective thermal diffusivity. Tested fluids were a glycerin/water mixture (Newtonian) and a CMC solution (pseudoplastic). Model was successfully adjusted and parameters were correlated with Reynolds number for flow.
\end{abstract}

\section{INTRODUCTION}

Non-isothermal laminar flow in tubes is a recurrent problem in many chemical and food processing applications, such as laminar flow reactors (LFR), tube sterilizers or tubular heat exchangers. A phenomenological model is required to represent flow, heat transfer, mass transfer and reaction in order to determine the distributions of temperature and concentration. In the case of corrugated or coiled tubes, the induced secondary flow improves mixing at low Reynolds number flow. However, modeling such systems can be rather complex and demand the use of CFD (computational fluid dynamics) tools to represent particular geometries and flow patterns (Bergles and Joshi, 1983; Barba et al., 2002; Solano et al., 2012; Pimenta and Campos, 2013).

The objective of this work is to present an alternative approach to model enhanced heat and mass transfer in LFR or heat exchangers. Besides coiled and corrugated tubes, heat and mass transfer augmentation are also associated with high relative wall roughness, mechanical vibration or the presence of fittings and curves. The vessel can be modeled as a straight smooth tube with developed laminar flow and the heat and mass transfer enhancement can be introduced in the model as an "effective" radial diffusivity that promotes mixing. This approach can lead to a good approximation of the heat and mass transfer in the vessel using a simpler mathematical model (Kechichian et al., 2012; Dantas et al., 2013). This would introduce two empirical parameters in the model to be determined through experiments: the effective radial diffusivity of heat $\alpha_{\text {eff }}$ and the effective radial diffusivity of mass $D_{\text {eff. }}$ The procedure to estimate these parameters is derived in this work and a case study is presented to validate this modeling approach. 


\section{MATHEMATICAL MODELING}

The Laminar Flow Reactor (LFR) or equivalent tubular system is modeled as a straight smooth tube with circular cross section, length $L$ and internal radius $R$. Average thermophysical properties of the fluid are considered. Single phase, incompressible, inelastic and developed flow is assumed with the following power-law velocity profile:

$$
v_{z}(r)=v_{m}\left(\frac{3 n+1}{n+1}\right)\left[1-\left(\frac{r}{R}\right)^{\frac{n+1}{n}}\right]
$$

where $v_{m}$ is the bulk velocity and $n$ is the flow behavior index; which is unitary for a Newtonian fluid (Chhabra and Richardson, 2008).

The model should take into account enhanced mass and heat transfer that are associated with corrugated tubes, coils, high relative wall roughness, mechanical vibration or the presence of fittings and curves. The empirical parameters for heat transfer $\left(\alpha_{e f f}\right)$ and mass transfer $\left(D_{\text {eff }}\right)$ can be determined through separate heat transfer and residence time distribution (RTD) experiments, respectively. Modeling for these two cases are derived as follows and parameter determination from experimental data is explained in Section 3.

\subsection{Heat Transfer Model}

For the experimental determination of the effective thermal diffusivity, a uniform wall temperature will be assumed for the modeling. In this case, the energy conservation in the LFR leads to the known Graetz problem for heat transfer in tube under laminar flow:

$$
v_{z} \frac{\partial T}{\partial z}-\alpha \frac{1}{r} \frac{\partial}{\partial r}\left(r \frac{\partial T}{\partial r}\right)=0
$$

where $T$ is the temperature and $\alpha$ is the thermal diffusivity. This equation takes into account the axial advection and the radial diffusion of heat under steady state conditions. Boundary conditions are: specification of the inlet temperature $T_{i n}$, symmetry at the center of the tube and specification of the wall temperature $T_{w}$ (Chhabra and Richardson, 2008).

By introducing the power-law velocity profile from Equation 1, Equation 2 can be expressed in dimensionless form:

$$
\left(\frac{3 n+1}{n+1}\right)\left(1-Y^{\frac{n+1}{n}}\right) \frac{\partial \Phi}{\partial Z}-\frac{1}{P e_{H}} \frac{1}{Y} \frac{\partial}{\partial Y}\left(Y \frac{\partial \Phi}{\partial Y}\right)=0
$$

where the dimensionless variables are $\Phi=\left(T-T_{w}\right) /\left(T_{i n}-T_{w}\right), Z=z / L$ and $Y=r / R$. Equation 3 depends on two parameters: the flow behavior index $n$ and the modified reactor Peclet number for enhanced heat transfer: 


$$
P e_{H}=\frac{L v_{m}}{\alpha_{e f f}}\left(\frac{R}{L}\right)^{2}
$$

The mixing cup average temperature along the tube $\Phi_{m}$ can be calculated as:

$$
\Phi_{m}(Z)=2\left(\frac{3 n+1}{n+1}\right) \int_{0}^{1}\left(1-Y^{\frac{n+1}{n}}\right) \Phi(Z, Y) Y d Y
$$

\subsection{Mass Transfer Model}

Information regarding mass diffusivity in the LFR can be determined through residence time distribution (RTD) experiments. To model the dispersion of a tracer along the vessel, a transient mass conservation equation is needed:

$$
\frac{\partial c_{t}}{\partial t}+v_{z} \frac{\partial c_{t}}{\partial z}-D_{t}\left[\frac{\partial^{2} c_{t}}{\partial z^{2}}+\frac{1}{r} \frac{\partial}{\partial r}\left(r \frac{\partial c_{t}}{\partial r}\right)\right]=0
$$

where $c_{t}(z, r, t)$ is the tracer concentration (Dantas et al., 2014). A step experiment is considered, where the tracer continuously enters the vessel with $c_{t}=c_{t, 0}$ and there is no tracer an $t=0$ (initial condition). Boundary conditions are: symmetry at the center of the tube, no diffusion at the tube wall and uniform inlet of tracer at the tube entrance. 1):

Equation 6 can be expressed in dimensionless form including the velocity profile (Equation

$$
\frac{\partial C}{\partial \theta}+\left(\frac{3 n+1}{n+1}\right)\left(1-Y^{\frac{n+1}{n}}\right) \frac{\partial C}{\partial Z}-\frac{1}{P e_{M}} \frac{1}{Y} \frac{\partial}{\partial Y}\left(Y \frac{\partial C}{\partial Y}\right)=0
$$

where the new dimensionless variables are $C=c_{t} / c_{t, 0}$ and $\theta=t / \tau$. Parameter $\tau=L / v_{m}$ is the characteristic time of the tube (ratio between internal volume and volumetric flow rate). The mass dispersion in the vessel is expressed through the modified reactor Peclet number for enhanced mass transfer:

$$
P e_{M}=\frac{L v_{m}}{D_{e f f}}\left(\frac{R}{L}\right)^{2}
$$

The mixing cup average concentration along the tube $C_{m}$ can be calculated as:

$$
C_{m}(Z, \theta)=2\left(\frac{3 n+1}{n+1}\right) \int_{0}^{1}\left(1-Y^{\frac{n+1}{n}}\right) C(Z, Y, \theta) Y d Y
$$




\section{MATERIALS AND METHODS}

For the experiments a laboratory scale thermal processing unit for liquid foods was used. This unit comprises two stainless steel double-pipe heat exchangers (heating and cooling) and a holding tube, as shown in Figure 1. Each heat exchanger has four straight tubes with an effective length of $1.675 \mathrm{~m}$ available for heat transfer. Internal and external diameters of the inner tube are 4.0 and $6.0 \mathrm{~mm}$. Internal and external diameters of the outer tube are 2.2 and $2.5 \mathrm{~cm}$. The heating section is connected to a pressurized hot water circuit (H.Cidade, Brazil) and the cooling section is connected to a $5.8 \mathrm{~kW}$ chiller (Mecalor, Brazil). An eccentric screw pump (Netzsch, Brazil) provides fluid flow and tested flow rates were 10, 20,30, 40 and $50 \mathrm{~L} / \mathrm{h}$. Tested fluids were a $80 / 20(\mathrm{w} / \mathrm{w})$ glycerin/water mixture (Newtonian) and a $1.0 \%(\mathrm{w} / \mathrm{w})$ solution of carboxymethylcellulose (CMC), which is pseudoplastic (Dantas et al., 2014). Generalized Reynolds number for power law fluids in tubes was calculated according to Carezzato et al. (2007). Physical properties of fluids were calculated at average temperature (Dantas et al., 2014).

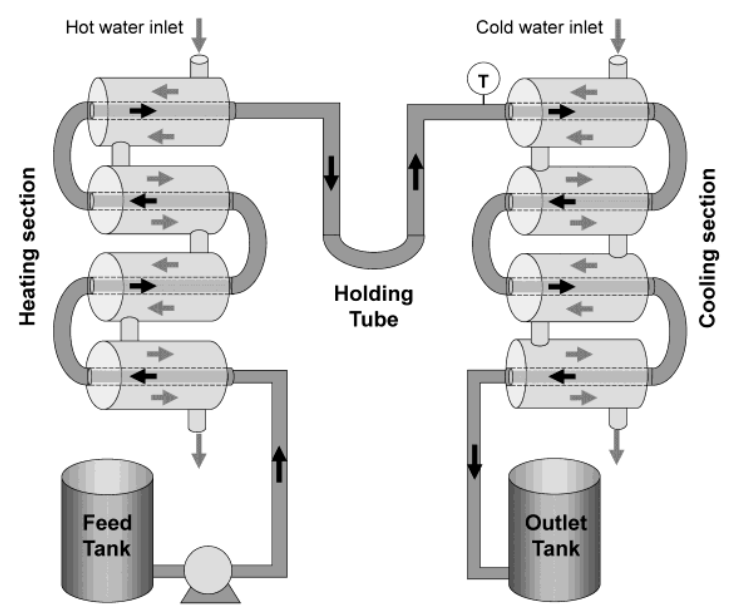

Figure 1 - Scheme of the thermal processing unit used in the experiments (Dantas et al., 2014)

\subsection{Heat Transfer Experiments}

For the heat transfer experiments, the cooling section of the thermal processing unit was used. The flow rate of the cooling water was kept at its maximum to provide a uniform wall temperature around the internal tube of the exchanger (the velocity in the annulus was approximately $9 \mathrm{~m} / \mathrm{s}$ ). The temperature of the external surface of the tube was assumed equal to the average temperature of the cooling water and the internal surface temperature was calculated based on the heat load of the exchanger and the thermal resistance of the tube wall.

Temperatures at the inlet and outlet of the exchanger were measured with inserted thermocouples and a data acquisition system (National Instruments, USA). Once steady state conditions were verified, temperatures were acquired for $30 \mathrm{~s}$ and average values calculated.

Equation (3) was used to simulate the heat transfer experiment. Numerical solution using a finite difference discretization method was implemented in software gPROMS 3.2 (PSE, UK). The outlet average temperature of the tube $\Phi_{m}(Z=1)$ was specified based on the experimental data and the Peclet number $P e_{H}$ was defined as a free variable to yield zero degrees of freedom. Obtained results were correlated with Reynolds number. 


\subsection{Mass Transfer Experiments}

For the RTD experiments, only one section of the holding tube was used with flow at room temperature $\left(21 \pm 1{ }^{\circ} \mathrm{C}\right)$. This section is equivalent to one hairpin of the heat exchanger with a total length of $3.64 \mathrm{~m}$ and internal volume of $45.7 \mathrm{~mL}$ (two straight tubes and a $180^{\circ}$ bend with $12.5 \mathrm{~cm}$ diameter). Injected tracer was $0.35 \mathrm{~mL}$ of $160 \mathrm{ppm}$ methylene blue in the test fluid, which was assessed using a spectrophotometer (Femto, Brazil) at $665 \mathrm{~nm}$ (absorbance was under 0.1). Outlet fluid was collected every 1 or $2 \mathrm{~s}$ and each sample was homogenized prior to assessment.

Five experiments were conducted for each flow rate. The mean residence time and the dimensionless E-curve $E_{\theta}(\theta)$ were determined according to Levenspiel (1989) and Fogler (2006). The active volume of the vessel $\left(V_{\text {active }}\right)$ was obtained from the plot of the mean residence time versus flow rate as $t_{m}=V_{\text {active }} l Q$. The active volume is smaller than the internal volume because of the presence of stagnation or recirculation areas, consequently, $t_{m} \leq \tau$.

In order to determine $P e_{M}$ for each run, the obtained experimental curve $E_{\theta}(\theta)$ was compared with the results from model simulation for different values of $P e_{M}$. The plot of the sum of squared errors on $E_{\theta}(\theta)$ versus $\log \left(P e_{M}\right)$ provided the modified Peclet with the minimum error. The adjusted values of $\mathrm{Pe}_{M}$ were correlated with Reynolds number.

The dispersion of the tracer was modeled through Equation 7, which was solved using a finite difference method in software gPROMS 3.2 (PSE, UK). Equation 9 for $Z=1$ (end of the tube) is actually the RTD F-curve: $F=C_{m}(Z=1, \theta)$. Consequently, the theoretical E-curve was numerically determined as $E_{\theta}(\theta)=\partial F / \partial \theta$.

\section{RESULTS AND DISCUSSION}

Figure 2 brings simulation results of the thermal model for a Newtonian fluid $(n=1)$. The effect of the modified Peclet number $P e_{H}$ on the heat transfer can be observed. Lower values of $P e_{H}$ imply on higher radial heat dispersion and, consequently, $\Phi_{m}(Z=1) \rightarrow 0.0$. It was verified that pseudo-plasticity slightly improved heat transfer, but he influence of the flow behavior index $n$ on the temperature distribution was small.
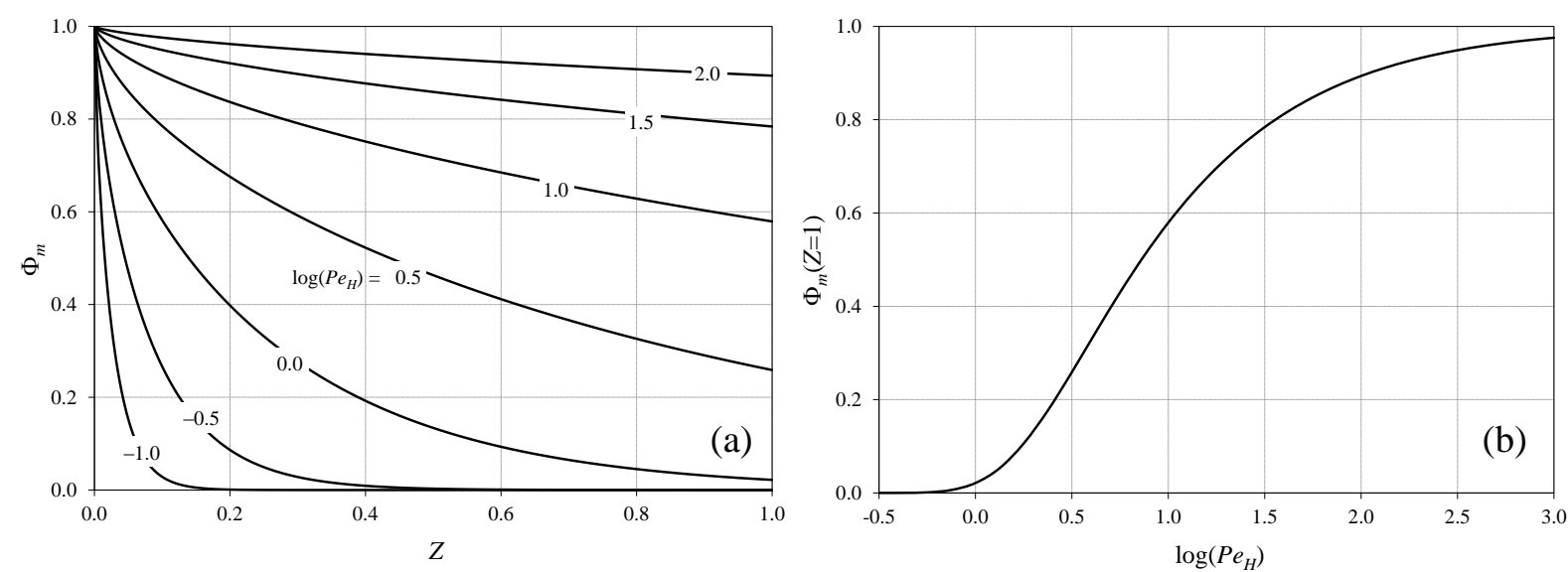

Figure 2 - Simulation results of the heat transfer model for $n=1$ and variable Peclet number $\left(P e_{H}\right)$ : a) Average temperature versus tube length $(Z)$; b) Outlet temperature versus $P e_{H}$. 
In order to determine $P e_{H}$ from experimental data, the average temperature must be known on at least one axial position $Z$ apart from the entrance. In this work, only the outlet temperature was measured. By specifying $\Phi_{m}(Z=1)$, the model simulation provided the corresponding value of $P e_{H}$. From Equation 4, the effective radial diffusivity of heat $\alpha_{\text {eff }}$ can be calculated. Thus, the improvement in heat transfer can be expressed by means of a heat transfer enhancement factor $F_{H}$ $=\alpha_{\text {eff }} / \alpha$. Figure 3 presents the obtained values of $F_{H}$ for the experiments with the 80/20 glycerin/water mixture $(n=1.0)$ and the $1.0 \%$ CMC solution ( $n$ between 0.43 and 0.47 , depending on average temperature). It can be seen that $F_{H}$ approaches unity for low Reynolds number and that $F_{H}$ improves with the flow rate because of the increased turbulence.
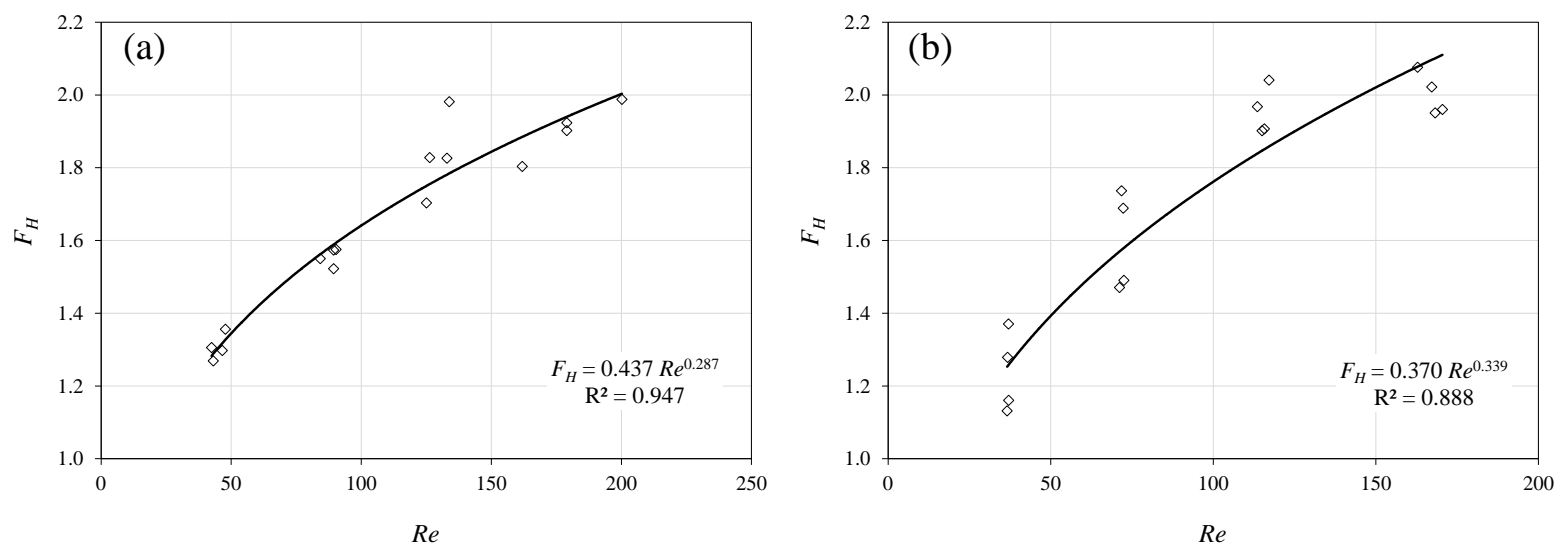

Figure 3 - Heat transfer enhancement factor versus Reynolds number for experiments with: a) $80 / 20$ Glycerin/water mixture; and b) $1.0 \%$ CMC solution.

In the experiments, the effective thermal diffusivity was up to two times higher than the fluid thermal diffusivity. This heat transfer enhancement can mainly be attributed to the high relative roughness of the tube wall (approximately 0.01). Moreover, the $180^{\circ}$ bends also contributed with the fluid mixing. Considering the uncertainty in the experimental data in Figure 3 , it is not possible to identity different behaviors between the two fluids.
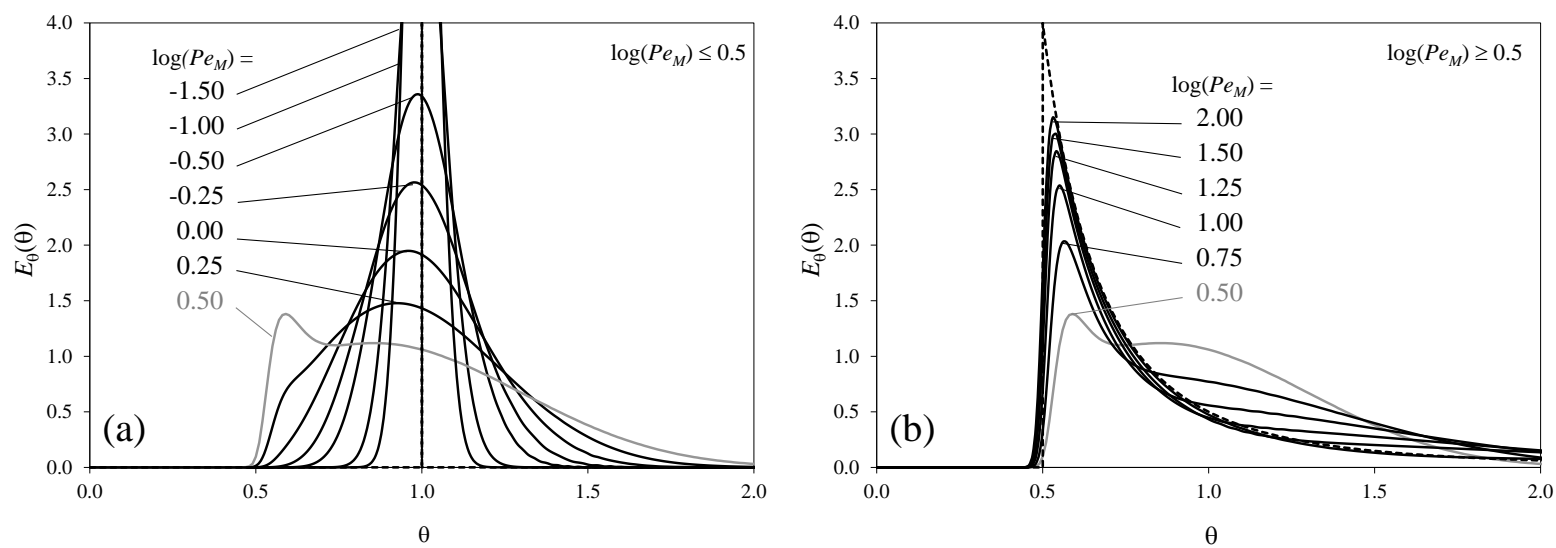

Figure 4 - Simulation results of the mass transfer model for $n=1$ and variable Peclet number $\left(P e_{M}\right)$ : a) E-curves for $\log \left(P e_{M}\right) \leq 0.5$; b) E-curves for $\log \left(P e_{M}\right) \geq 0.5$ (Dantas et al., 2014). 
Figure 4 presents simulation results of the mass transfer model for a Newtonian fluid $(n=$ 1) in the form of theoretical E-curves for the dispersion of a tracer. Results were split in two figures because two distinct behaviors were observed. For $\log \left(P e_{M}\right) \leq 0.5$, the E-curve shows a peak near $\theta=1.0$ that is characteristic of turbulent flow (high dispersion). On the other hand, for $\log \left(P e_{M}\right) \geq 0.5$, the E-curve shows a peak around $\theta=0.5$ that is characteristic of laminar flow with low radial dispersion. Pseudo-plasticity narrowed the tracer dispersion because the minimum residence time was higher than $\theta=0.5$ (Newtonian). The limiting case in Figure 4(b) (dashed line) corresponds to segregated laminar flow. For $\log \left(P e_{M}\right) \approx 0.5$, the velocity profile and the radial dispersion play important roles on the tracer dispersion and the E-curve has two peaks.

The E-curves obtained from the experiments in the equipment were compared to the model simulation results and the error was minimized in order to determine the corresponding value of the modified Peclet $P e_{M}$. Results are presented in Figure 5 for the experiments with the 80/20 glycerin/water mixture $(n=1.0)$ and the $1.0 \% \mathrm{CMC}$ solution $\left(n=0.4\right.$ at $\left.T=21^{\circ} \mathrm{C}\right)$ as the effective radial mass diffusivity versus Reynolds number. Considering the uncertainty on the diffusivities, a linear correlation could be adjusted and similar behavior was observed for both fluids. Average values of the Modified Peclet number were $\log \left(P e_{M}\right)=0.26$ for the glycerin/water mixture and $\log \left(P e_{M}\right)=0.52$ for the CMC solution. From Figure 4 it can be concluded that radial diffusion was important to the dispersion of the tracer for these values of $\mathrm{Pe}_{M}$.
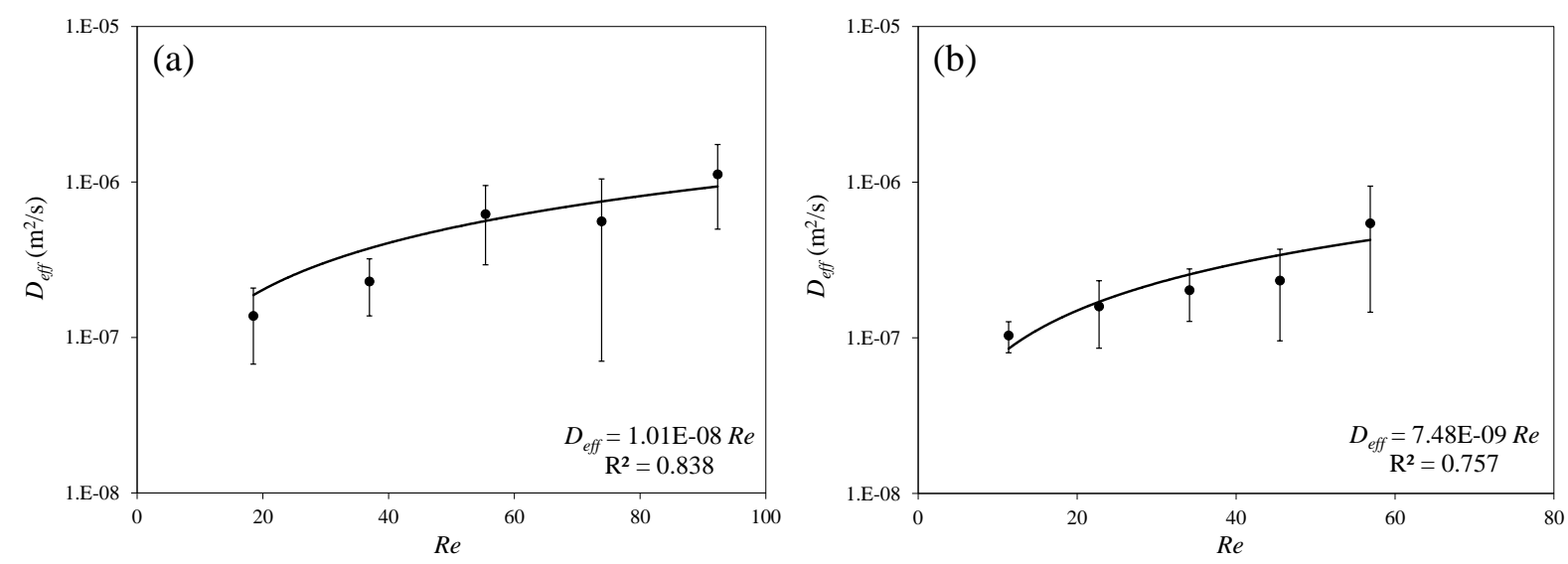

Figure 5 - Effective radial mass diffusivity versus Reynolds number for experiments with: a) 80/20 Glycerin/water mixture; and b) $1.0 \%$ CMC solution.

Another import result that was obtained from the RTD experiments is the active volume of the vessel. The adjusted values from the glycerin/water experiments and the CMC solution experiments were $91 \%$ and $89 \%$, respectively. This indicates that approximately $10 \%$ of the internal volume of the tube is related with stagnation or recirculation zones or the presence of deposits at the tube wall. Since the equipment was used for thermal processing of liquid foods, fooling in the holding tube is a plausible explanation.

\section{CONCLUSIONS}

Two parameters were introduced in the Laminar Flow Reactor (LFR) model in order to simulate enhanced heat and mass transfer: the effective radial diffusivity of heat $\alpha_{\text {eff }}$ and the effective radial diffusivity of mass $D_{\text {eff. }}$ Through heat transfer and RTD experiments, it was 


\section{9 a 22 de outubro de 2014 \\ Florianópolis/SC}

possible to estimate these parameters for a liquid food thermal processing unit that has a high relative roughness on the tube wall. For this case study, the adjusted parameters can be applied to simulate the temperature distribution and the microbial inactivation of the process using the model developed by Kechichian et al. (2012). This modeling approach of the LFR provides a simple form to take into account heat and/or mass transfer enhancement in tubular laminar flow problems.

\section{ACKNOWLEDGEMENTS}

The authors would like to acknowledge the financial support from the São Paulo Research Foundation (FAPESP) under grant 2009/07934-3.

\section{REFERENCES}

BARBA, A., RAINIERI, S.; SPIGA, M. Heat transfer enhancement in a corrugated tube. Int. Commun. Heat Mass Tran., 29(3), 313-322, 2002.

BERGLES, A.E.; JOSHI, S.D. Augmentation techniques for low Reynolds number in-tube flow. In: KAKAÇ, S., SHAH, R.K.; BERGLES, A.E. (Eds.), Low Reynolds Number Flow Heat Exchangers. Washington: Hemisphere, 1983.

CAREZZATO, A.; ALCANTARA, M.R.; TELIS-ROMERO, J.; TADINI, C.C.; GUT, J.A.W. Non-Newtonian heat transfer on a plate heat exchanger with generalized configurations. Chem. Eng. Technol., 30(1), 21-26, 2007.

CHHABRA, R.P.; RICHARDSON, J.F. Non-Newtonian Flow and Applied Rheology: Engineering Applications, $2^{\text {nd }}$ ed. Butterworth-Heinemann, 2008.

DANTAS, J.A.T.A.; GUT, J.A.W.; PEGORARO, P.R. Determination of the effective radial mass diffusivity in tubular reactors under non-Newtonian laminar flow using residence time distribution data. Int. J. Heat Mass Tran., 71, 18-25, 2014.

FOGLER, H.S. Elements of Chemical Reaction Engineering, $4^{\text {th }}$ ed. Upper Saddle River: Prentice Hall, 2006.

KECHICHIAN, V.; CRIVELLARI, G.P.; GUT, J.A.W.; TADINI, C.C. Modeling of continuous thermal processing of a non-newtonian liquid food under diffusive laminar flow in a tubular system. Int. J. Heat Mass Tran., 55(21-22), 5783-5792, 2012.

LEVENSPIEL, O. The Chemical Reactor Omnibook. Corvallis: OSU, 1989.

PIMENTA, T.A.; CAMPOS, J.B.L.M., Heat transfer coefficients from Newtonian and nonNewtonian fluids flowing in laminar regime in a helical coil. Int. J. Heat Mass Tran., 58(12), 676-690, 2013.

SOLANO, J.P.; HERRERO, R.; ESPÍN, S.; PHAN, A.N.; HARVEY, A.P. Numerical study of the flow pattern and heat transfer enhancement in oscillatory baffled reactors with helical coil inserts, Chem. Eng. Res. Des., 90(6), 732-742, 2012. 